\title{
A Canonical Definition of Shape
}

\author{
Davy Paindaveine* \\ Université Libre de Bruxelles
}

\begin{abstract}
Very general concepts of scatter, extending the traditional notion of covariance matrices, have become classical tools in robust multivariate analysis. In many problems of practical importance (principal components, canonical correlation, testing for sphericity), only homogeneous functions of the scatter matrix are of interest. In line with this fact, scatter functionals often are only defined up to a positive scalar factor, yielding a family of scatter matrices rather than a uniquely defined one. In such families, it is natural to single out one representative by imposing a normalization constraint: this normalized scatter is called a shape matrix. In the particular case of elliptical families, this constraint in turn induces a concept of scale; along with a location center and a standardized radial density, the shape and scale parameters entirely characterize an elliptical density. In this paper, we show that one and only normalization has the additional properties that (i) the resulting Fisher information matrices for shape and scale, in locally asymptotically normal (LAN) elliptical families, are block-diagonal, and that (ii) the semiparametric elliptical families indexed by location, shape, and completely unspecified radial densities are adaptive. This particular normalization, which imposes that the determinant of the shape matrix be equal to one, therefore can be considered canonical.
\end{abstract}

Key words and phrases: Elliptic densities; Scatter matrix; Shape matrix; Local asymptotic normality; Semiparametric efficiency; Adaptivity

\section{Introduction.}

The multivariate concepts of location and scatter, extending to the multivariate context the traditional concepts of location and scale are generally characterized via their behavior under affine transformations of the observation space. More precisely, denoting by $\mathbf{X}$ a $k$-variate random vector with probability distribution $P^{\mathbf{X}}$, consider a couple $(\boldsymbol{\theta}, \boldsymbol{\Sigma})$ of functionals defined over $\left\{P^{\mathbf{A X}+\mathbf{b}}: \mathbf{A}\right.$ an invertible $k \times k$ real matrix, $\left.\mathbf{b} \in \mathbb{R}^{k}\right\}$ mapping $P^{\mathbf{X}}$ onto $\left(\boldsymbol{\theta}^{\mathbf{X}}, \boldsymbol{\Sigma}^{\mathbf{X}}\right) \in \mathbb{R}^{k} \times \mathcal{S}_{k}$, where $\mathcal{S}_{k}$ denotes the set of symmetric positive definite real $k \times k$ matrices - throughout, $k \geq 2$. This couple is called a location-scatter functional iff

$$
\left(\boldsymbol{\theta}^{\mathbf{A X}+\mathbf{b}}, \Sigma^{\mathbf{A X}+\mathbf{b}}\right)=\left(\mathbf{A} \boldsymbol{\theta}^{\mathbf{X}}+\mathbf{b}, \mathbf{A} \Sigma^{\mathbf{X}} \mathbf{A}^{\prime}\right)
$$

for any invertible $k \times k$ matrix $\mathbf{A}$ and any $\mathbf{b} \in \mathbb{R}^{k}$. The traditional example of such a couple of course is the mean and the covariance matrix, but many other solutions exist, and the problem of defining robust counterparts to means and covariances has generated

*Davy Paindaveine, Institut de Recherche en Statistique, ECARES, and Département de Mathématique, Université Libre de Bruxelles, Avenue F.D. Roosevelt, 50, CP 114, B-1050 Bruxelles, Belgium. The author is also member of ECORE, the recently created association between CORE and ECARES. 
a huge literature which we do not attempt to review here - see Maronna et al. (2006) or Zuo (2006) for recent surveys.

In many problems in multivariate analysis, it is sufficient to know - or to estimatenormalized versions of scatter matrices to be able to perform the analysis (see below). In line with this fact, scatter matrices often are only defined up to a positive factorsee, for instance, Tyler $(1983,1987)$. In such families of scatter matrices, it is natural to pick out one representative by imposing a normalization constraint. More specifically, let $S: \mathcal{S}_{k} \rightarrow \mathbb{R}_{0}^{+}$be a 1-homogeneous function-i.e., satisfying $S(\lambda \boldsymbol{\Sigma})=\lambda S(\boldsymbol{\Sigma})$ for all $\lambda>0$-and define $\mathcal{V}_{k}^{S}:=\left\{\mathbf{V} \in \mathcal{S}_{k}: S(\mathbf{V})=1\right\}$ : the elements of $\mathcal{V}_{k}^{S}$ are called shape matrices, and $\mathbf{V}_{S}^{\mathbf{X}}:=\boldsymbol{\Sigma}^{\mathbf{X}} / S\left(\boldsymbol{\Sigma}^{\mathbf{X}}\right)$ is called the shape matrix of $\mathbf{X}$; the latter clearly is a 0-homogeneous (in the sequel, we simply write homogeneous) function of $\boldsymbol{\Sigma}^{\mathbf{X}}$, in the sense that all scatter matrices $\lambda \boldsymbol{\Sigma}^{\mathbf{X}}, \lambda>0$ yield the same shape matrix.

The choice of $S$ is arbitrary and, to some extent, inessential (see the comments below). Classical choices include

(i) $S(\boldsymbol{\Sigma})=\Sigma_{11}$ (Hallin and Paindaveine (2006a), Hallin et al. (2006), Hettmansperger and Randles (2002), and Randles (2000)),

(ii) $S(\boldsymbol{\Sigma})=(\operatorname{tr} \boldsymbol{\Sigma}) / k$ (Dümbgen (1998), Ollila et al. (2004), and Tyler (1987)), and

(iii) $S(\boldsymbol{\Sigma})=|\boldsymbol{\Sigma}|^{1 / k}$ (Dümbgen and Tyler (2005), Hallin and Paindaveine (2008a), SalibianBarrera et al. (2006), Taskinen et al. (2006), and Tatsuoka and Tyler (2000)).

Now consider the particular case of a $k$-variate elliptical random vector $\mathbf{X}$, that is, letting $d(\mathbf{x}, \boldsymbol{\theta} ; \boldsymbol{\Sigma}):=\left((\mathbf{x}-\boldsymbol{\theta})^{\prime} \boldsymbol{\Sigma}^{-1}(\mathbf{x}-\boldsymbol{\theta})\right)^{1 / 2}$ and denoting by $|\mathbf{M}|$ the determinant of the square matrix $\mathbf{M}$, assume that $P^{\mathbf{X}}$ admits the density

$$
\mathbf{x} \mapsto \frac{c_{k, f_{1}}}{|\boldsymbol{\Sigma}|^{1 / 2}} f_{1}(d(\mathbf{x}, \boldsymbol{\theta} ; \boldsymbol{\Sigma})),
$$

where $\boldsymbol{\theta}$, the center of symmetry, is a $k$-dimensional real vector, $\boldsymbol{\Sigma}$ belongs to $\mathcal{S}_{k}$, and $f_{1}$ : $\mathbb{R}_{0}^{+} \longrightarrow \mathbb{R}_{0}^{+}$, the standardized radial density is such that $\mu_{k-1, f_{1}}<\infty$, with $\mu_{\ell, f_{1}}:=$ $\int_{0}^{\infty} r^{\ell} f_{1}(r) d r\left(c_{k, f_{1}}\right.$ is a normalization factor). To ensure identifiability of $\boldsymbol{\Sigma}$ and $c_{k, f_{1}} \times f_{1}$ without imposing any moment conditions, the pdf of $d(\mathbf{X}, \boldsymbol{\theta} ; \boldsymbol{\Sigma})$ under (2) (that is, $r \mapsto$ $\left.\tilde{f}_{1 k}(r):=\left(\mu_{k-1, f_{1}}\right)^{-1} r^{k-1} f_{1}(r) I_{[r>0]}\right)$ is assumed to have median one.

Under this elliptical setting, the only solutions of (1) are the couples $(\boldsymbol{\theta}, \lambda \boldsymbol{\Sigma})$, with arbitrary $\lambda>0$. It follows that the shape $\mathbf{V}_{S}=\boldsymbol{\Sigma} / S(\boldsymbol{\Sigma})$ is uniquely defined for any 1-homogeneous function $S$, and that $\sigma_{S}:=(S(\boldsymbol{\Sigma}))^{1 / 2}$, as the median of $d\left(\mathbf{X}, \boldsymbol{\theta} ; \mathbf{V}_{S}\right)$, has the interpretation of a scale parameter. This allows for rewriting (2) as

$$
\mathbf{x} \mapsto \frac{c_{k, f_{1}}}{\sigma_{S}^{k}\left|\mathbf{V}_{S}\right|^{1 / 2}} f_{1}\left(\frac{1}{\sigma_{S}} d\left(\mathbf{x}, \boldsymbol{\theta} ; \mathbf{V}_{S}\right)\right)=: \frac{c_{k, f}}{\left|\mathbf{V}_{S}\right|^{1 / 2}} f\left(d\left(\mathbf{x}, \boldsymbol{\theta} ; \mathbf{V}_{S}\right)\right)
$$

This latter density is indexed by $\boldsymbol{\theta}, \mathbf{V}_{S}$, and the (non-standardized) radial density $f$. Under finite second-order moments, of course, $\boldsymbol{\Sigma}$ reduces to a multiple of $\operatorname{Cov}[\mathbf{X}]$, and hence $\mathbf{V}_{S}=\operatorname{Cov}[\mathbf{X}] / S(\operatorname{Cov}[\mathbf{X}])$.

Whatever the choice of $S$, the shape matrix $\mathbf{V}_{S}$ is a parameter of primary interest in a number of standard problems in multivariate analysis. Principal component analysis (PCA), canonical correlation analysis (CCA), and the problem of testing for sphericity, among others, only depend on shape - rather than on scatter or covariance matrices; see, 
for instance, Croux and Haesbroeck (2000), Hallin and Paindaveine (2006a), and Taskinen et al. (2006). Inference on shape is thus an essential issue in the domain.

The choice of $S$ so far is still arbitrary. The objective of this paper is to show that decision-theoretic arguments, involving the structure of Fisher information and semiparametric efficiency, strongly suggest adopting the determinant-based normalization $S(\boldsymbol{\Sigma})=$ $|\boldsymbol{\Sigma}|^{1 / k}$. This particular choice indeed is the only one for which

(a) the Fisher information matrices for scale and shape, in locally asymptotically normal (LAN) elliptical families, are block-diagonal, and

(b) the semiparametric elliptical families indexed by location $\boldsymbol{\theta}$, shape $\mathbf{V}_{S}$, and completely unspecified radial densities $f$ (see (3)) are adaptive; this adaptivity result is much stronger than the one established in Bickel (1982) (see Section 2 for a discussion).

These two properties considerably simplify the structure of information, and in principle allow for parametrically efficient inference for shape, under unspecified $(\boldsymbol{\theta}, f)$ (equivalently, unspecified $\left.\left(\boldsymbol{\theta}, \sigma_{S}, f_{1}\right)\right)$. The determinant-based concepts of shape and scale therefore can be considered canonical.

The paper is organized as follows. In Section 2, we list and discuss the assumptions that are needed in the sequel, and state, for an arbitrary normalization $S$, the local and asympotic normality (LAN) property of elliptical families. Section 3 states the main result of the paper and discusses some of its implications. Finally, the proofs are given in Section 4.

\section{Assumptions, notation, and local asymptotic normality.}

The following notation will be used. For any $k \times k$ matrix $\mathbf{A}$, let vec $\mathbf{A}$ be the $k^{2}$-vector resulting from stacking the columns of $\mathbf{A}$ on top of each other. If $\mathbf{A}$ moreover is symmetric, write vech $\mathbf{A}:=\left(A_{11},(\operatorname{vech} \mathbf{A})^{\prime}\right)^{\prime}$ for the $(K+1)$-vector (throughout, $K=k(k+1) / 2-1$ ) obtained by stacking the upper-triangular elements of $\mathbf{A}=\left(A_{i j}\right)$ : vech $\mathbf{A}$ thus stands for vech $\mathbf{A}$ deprived of its first component $A_{11}$. On the scale functional $S$ we make the following assumption.

Assumption (A1). The scale functional $S: \mathcal{S}_{k} \rightarrow \mathbb{R}_{0}^{+}$(i) is 1-homogeneous (see Section 1), (ii) is differentiable, with $\frac{\partial S}{\partial \Sigma_{11}}(\boldsymbol{\Sigma}) \neq 0$ for all $\boldsymbol{\Sigma} \in \mathcal{S}_{k}$, and (iii) satisfies $S\left(\mathbf{I}_{k}\right)=1$, where $\mathbf{I}_{k}$ denotes the $k$-dimensional identity matrix.

Clearly, one can also look at $\boldsymbol{\Sigma} \mapsto S(\boldsymbol{\Sigma})$ as a function of vech $\boldsymbol{\Sigma}$ : with a slight abuse of notation, we indifferently write $S(\boldsymbol{\Sigma})$ or $S(\operatorname{vech} \boldsymbol{\Sigma})$ in the sequel, and denote by $\nabla S(\operatorname{vech} \boldsymbol{\Sigma})$ the gradient $\operatorname{grad}_{\operatorname{vech} \Sigma} S(\operatorname{vech} \boldsymbol{\Sigma})$. Under Assumption $(\mathrm{A} 1),\left(\mathbf{V}_{S}\right)_{11}$, for any $\mathbf{V}_{S} \in \mathcal{V}_{k}^{S}$, can be recovered from (vech $\mathbf{V}_{S}$ ). Also note that the special role of $\Sigma_{11}$ in Assumption (A1) could have been played by any other entry of $\boldsymbol{\Sigma}$. Assuming that some other component of $\nabla S$ is non-zero would allow, for instance, for dealing with scale functionals such as $S(\boldsymbol{\Sigma})=$ $\Sigma_{22}$ or $S(\boldsymbol{\Sigma})=\left(\prod_{i=2}^{k} \Sigma_{i i}\right)^{1 /(k-1)}$ — with appropriate redefinition of the vech operator. As the extension of our results to such cases is trivial, we stick to Assumption (A1) in the sequel.

Denote by $P_{\boldsymbol{\theta}, \boldsymbol{\Sigma}, f_{1}}^{n}$ or equivalently (for any given $S$ ) $P_{\boldsymbol{\theta}, \sigma_{S}^{2}, \mathbf{V}_{S}, f_{1}}$ the distribution of an i.i.d. $n$-tuple $\left(\mathbf{X}_{1}, \ldots, \mathbf{X}_{n}\right)$ with density (2) or (3). For given $S$ satisfying Assumption (A1), the 
scatter parameter $\boldsymbol{\Sigma}$ (in vector form, vech $\boldsymbol{\Sigma}$ ) decomposes into scale and shape parameters through $\boldsymbol{\Sigma}=\sigma_{S}^{2} \mathbf{V}_{S}$, where $\sigma_{S}^{2}:=S(\boldsymbol{\Sigma})$ and $\mathbf{V}_{S}:=\boldsymbol{\Sigma} / S(\boldsymbol{\Sigma}) \in \mathcal{V}_{k}^{S}$. In vector form, dropping $\left(\mathbf{V}_{S}\right)_{11}$, the new parameter is thus $\left.\boldsymbol{\vartheta}_{S}:=\left(\boldsymbol{\theta}^{\prime}, \sigma_{S}^{2} \text {, (vech } \mathbf{V}_{S}\right)^{\prime}\right)^{\prime} \in \boldsymbol{\Theta}_{S}\left(:=\mathbb{R}^{k} \times \mathbb{R}_{0}^{+} \times \operatorname{vech} \mathcal{V}_{k}^{S}\right)$. Theorem 2.1 below states that, under mild regularity conditions on $f_{1}$, the families of distributions $\mathcal{P}_{S ; f_{1}}^{n}:=\left\{P_{\vartheta_{S}, f_{1}}^{n}: \boldsymbol{\vartheta}_{S} \in \boldsymbol{\Theta}_{S}\right\}$ are locally asymptotically normal (LAN; see Le Cam 1986). This theorem extends to an arbitrary scale functional $S$ the result obtained for $S(\boldsymbol{\Sigma})=\Sigma_{11}$ in Hallin and Paindaveine (2006a), where minimal assumptions are given; here, for the sake of simplicity, we rather provide the following sufficient one.

Assumption (A2). The standardized radial density $f_{1}$ belongs to the collection $\mathcal{F}$ of absolutely continuous functions, with a.e.-derivative $\dot{f}_{1}$, and, letting $\varphi_{f_{1}}:=-\dot{f}_{1} / f_{1}$, the quantities $\mathcal{I}_{k}\left(f_{1}\right):=\int_{0}^{\infty}\left(\varphi_{f_{1}}(r)\right)^{2} \tilde{f}_{1 k}(r) d r$ and $\mathcal{J}_{k}\left(f_{1}\right):=\int_{0}^{\infty} r^{2}\left(\varphi_{f_{1}}(r)\right)^{2} \tilde{f}_{1 k}(r) d r$ are finite.

The finiteness of the radial Fisher informations for location $\mathcal{I}_{k}\left(f_{1}\right)$ and scale/shape $\mathcal{J}_{k}\left(f_{1}\right)$ does not imply any moment conditions. Hence, Assumption (A2) is extremely mild and turns out to be satisfied at Gaussian densities as well as at all Student and powerexponential densities (see Hallin and Paindaveine 2006a).

The following notation is needed in the statement of LAN. Denoting by $\mathbf{e}_{\ell}$ the $\ell$ th vector of the canonical basis of $\mathbb{R}^{k}$, let $\mathbf{K}_{k}:=\sum_{i, j=1}^{k}\left(\mathbf{e}_{i} \mathbf{e}_{j}^{\prime}\right) \otimes\left(\mathbf{e}_{j} \mathbf{e}_{i}^{\prime}\right)$ be the $k^{2} \times k^{2}$ commutation matrix, and put $\mathbf{J}_{k}:=\left(\operatorname{vec} \mathbf{I}_{k}\right)\left(\operatorname{vec} \mathbf{I}_{k}\right)^{\prime}$. Write also $\mathbf{A}^{\otimes 2}$ for the Kronecker product $\mathbf{A} \otimes \mathbf{A}$. Finally, for any $\boldsymbol{\Sigma} \in \mathcal{S}_{k}$ and $S$ satisfying (A1), let $\mathbf{M}_{S}^{\boldsymbol{\Sigma}}:=\mathbf{M}_{S, k}^{\boldsymbol{\Sigma}}$ be the $K \times k^{2}$ matrix such that $\left(\mathbf{M}_{S}^{\Sigma}\right)^{\prime}($ vech $\mathbf{v})=\operatorname{vec} \mathbf{v}$ for any symmetric $k \times k$ matrix $\mathbf{v}$ satisfying $(\nabla S(\operatorname{vech} \boldsymbol{\Sigma}))^{\prime}(\operatorname{vech} \mathbf{v})=0$. Note that $(\nabla S(\operatorname{vech} \boldsymbol{\Sigma}))^{\prime}(\operatorname{vech} \mathbf{v})=0$ holds for $S(\boldsymbol{\Sigma})=\Sigma_{11}$, $S(\boldsymbol{\Sigma})=(\operatorname{tr} \boldsymbol{\Sigma}) / k$, and $S(\boldsymbol{\Sigma})=|\boldsymbol{\Sigma}|^{1 / k}$, iff $(\mathbf{v})_{11}=0, \operatorname{tr} \mathbf{v}=0$, and $\operatorname{tr}\left(\boldsymbol{\Sigma}^{-1} \mathbf{v}\right)=0$, respectively.

Theorem 2.1 Under Assumptions (A1) and (A2), the family $\mathcal{P}_{S ; f_{1}}^{n}=\left\{P_{\boldsymbol{\vartheta}_{S}, f_{1}}^{n}: \boldsymbol{\vartheta}_{S} \in \boldsymbol{\Theta}_{S}\right\}$ is LAN. More precisely, for any $\left.\boldsymbol{\vartheta}_{S}=\left(\boldsymbol{\theta}^{\prime}, \sigma_{S}^{2} \text {, (vech } \mathbf{V}_{S}\right)^{\prime}\right)^{\prime}$ and any bounded sequence $\boldsymbol{\tau}_{n} \in$ $\mathbb{R}^{k+K+1}$, we have that (i) under $P_{\boldsymbol{\vartheta}_{S}, f_{1}}^{n}$,

$$
\log \left(d P_{\boldsymbol{\vartheta}_{S}+n^{-1 / 2} \boldsymbol{\tau}_{n}, f_{1}}^{n} / d P_{\boldsymbol{\vartheta}_{S}, f_{1}}^{n}\right)=\boldsymbol{\tau}_{n}^{\prime} \boldsymbol{\Delta}_{\boldsymbol{\vartheta}_{S}, f_{1}}^{n}-\frac{1}{2} \boldsymbol{\tau}_{n}^{\prime} \boldsymbol{\Gamma}_{\boldsymbol{\vartheta}_{S}, f_{1}} \boldsymbol{\tau}_{n}+o_{P}(1)
$$

where, letting $d_{i}:=d\left(\mathbf{X}_{i}, \boldsymbol{\theta} ; \mathbf{V}_{S}\right)$ and $\mathbf{U}_{i}:=\mathbf{V}_{S}^{-1 / 2}\left(\mathbf{X}_{i}-\boldsymbol{\theta}\right) / d_{i} \quad$ (throughout, $\mathbf{V}_{S}^{1 / 2}$ is taken symmetric $), \boldsymbol{\Delta}_{\vartheta_{S}, f_{1}}^{n}:=\left(\left(\boldsymbol{\Delta}_{\vartheta_{S}, f_{1} ; 1}^{n}\right)^{\prime}, \Delta_{\vartheta_{S}, f_{1} ; 2}^{n},\left(\boldsymbol{\Delta}_{\vartheta_{S}, f_{1} ; 3}^{n}\right)^{\prime}\right)^{\prime}$, with

and

$$
\begin{gathered}
\Delta_{\vartheta_{S}, f_{1} ; 1}^{n}:=\frac{1}{\sigma_{S} \sqrt{n}} \sum_{i=1}^{n} \varphi_{f_{1}}\left(\frac{d_{i}}{\sigma_{S}}\right) \mathbf{V}_{S}^{-1 / 2} \mathbf{U}_{i}, \\
\Delta_{\vartheta_{S}, f_{1} ; 2}^{n}:=\frac{1}{2 \sigma_{S}^{2} \sqrt{n}} \sum_{i=1}^{n}\left(\varphi_{f_{1}}\left(\frac{d_{i}}{\sigma_{S}}\right) \frac{d_{i}}{\sigma_{S}}-k\right),
\end{gathered}
$$

$$
\Delta_{\vartheta_{S}, f_{1} ; 3}^{n}:=\frac{1}{2 \sqrt{n}} \mathbf{M}_{S}^{\mathbf{V}_{S}}\left(\mathbf{V}_{S}^{\otimes 2}\right)^{-1 / 2} \sum_{i=1}^{n} \operatorname{vec}\left(\varphi_{f_{1}}\left(\frac{d_{i}}{\sigma_{S}}\right) \frac{d_{i}}{\sigma_{S}} \mathbf{U}_{i} \mathbf{U}_{i}^{\prime}-\mathbf{I}_{k}\right),
$$

and that (ii) the central sequence $\Delta_{\vartheta_{S}, f_{1}}^{n}$, still under $P_{\vartheta_{S}, f_{1}}^{n}$, is asymptotically normal with mean zero and covariance matrix

$$
\Gamma_{\vartheta_{S}, f_{1}}:=\left(\begin{array}{ccc}
\Gamma_{\boldsymbol{\vartheta}_{S}, f_{1} ; 11} & \mathbf{0} & \mathbf{0} \\
\mathbf{0} & \Gamma_{\vartheta_{S}, f_{1} ; 22} & \Gamma_{\vartheta_{S}, f_{1} ; 32}^{\prime} \\
\mathbf{0} & \boldsymbol{\Gamma}_{\boldsymbol{\vartheta}_{S}, f_{1} ; 32} & \boldsymbol{\Gamma}_{\vartheta_{S}, f_{1} ; 33}
\end{array}\right)
$$


with

$$
\begin{gathered}
\Gamma_{\vartheta_{S}, f_{1} ; 11}:=\frac{\mathcal{I}_{k}\left(f_{1}\right)}{k \sigma_{S}^{2}} \mathbf{V}_{S}^{-1} \\
\Gamma_{\boldsymbol{\vartheta}_{S}, f_{1} ; 22}:=\frac{\mathcal{J}_{k}\left(f_{1}\right)-k^{2}}{4 \sigma_{S}^{4}}, \quad \Gamma_{\boldsymbol{\vartheta}_{S}, f_{1} ; 32}:=\frac{\mathcal{J}_{k}\left(f_{1}\right)-k^{2}}{4 k \sigma_{S}^{2}} \mathbf{M}_{S}^{\mathbf{V}_{S}}\left(\operatorname{vec} \mathbf{V}_{S}^{-1}\right),
\end{gathered}
$$

and

$$
\Gamma_{\vartheta_{S}, f_{1} ; 33}:=\frac{1}{4} \mathbf{M}_{S}^{\mathbf{V}_{S}}\left(\mathbf{V}_{S}^{\otimes 2}\right)^{-1 / 2}\left[\frac{\mathcal{J}_{k}\left(f_{1}\right)}{k(k+2)}\left(\mathbf{I}_{k^{2}}+\mathbf{K}_{k}+\mathbf{J}_{k}\right)-\mathbf{J}_{k}\right]\left(\mathbf{V}_{S}^{\otimes 2}\right)^{-1 / 2}\left(\mathbf{M}_{S}^{\mathbf{V}_{S}}\right)^{\prime} .
$$

The block-diagonal structure of the information matrix (6) implies that the nonspecification of the location centre $\boldsymbol{\theta}$ does not affect optimal parametric performances when estimating $\mathbf{V}_{S}$ and/or $\sigma_{S}^{2}$, or when performing tests about the same; more precisely, when estimating $\mathbf{V}_{S}$ for instance, the optimal asymptotic covariance matrix that can be achieved (at $P_{\boldsymbol{\theta}, \sigma_{S}^{2}}^{n}, \mathbf{V}_{S}, f_{1}$ ) by an estimator of $\mathbf{V}_{S}$ is the same in $\mathcal{P}_{S ; \sigma_{S}^{2}, f_{1}}^{n}:=\left\{P_{\boldsymbol{\theta}, \sigma_{S}^{2}, \mathbf{V}_{S}, f_{1}}\right.$ : $\left.\boldsymbol{\theta} \in \mathbb{R}^{k}, \mathbf{V}_{S} \in \mathcal{V}_{k}^{S}\right\}$ as in $\mathcal{P}_{S ; \boldsymbol{\theta}, \sigma_{S}^{2}, f_{1}}^{n}:=\left\{P_{\boldsymbol{\theta}, \sigma_{S}^{2}, \mathbf{V}_{S}, f_{1}}^{n}: \mathbf{V}_{S} \in \mathcal{V}_{k}^{S}\right\}$, where $\boldsymbol{\theta}$ is specified, and is actually given by $\left(\boldsymbol{\Gamma}_{\vartheta_{S}, f_{1} ; 33}\right)^{-1}$. Since the latter does only depend on $\mathbf{V}_{S}$ and $f_{1}$, so does this optimal performance.

On the contrary, the non-zero covariance between the scale and shape parts of the central sequences implies that, when estimating $\mathbf{V}_{S}$, the non-specification of $\sigma_{S}^{2}$ affects the optimal parametric performance at $f_{1}$. The latter actually is given by the $f_{1}$-efficient Fisher information for shape

$$
\begin{aligned}
\boldsymbol{\Gamma}_{\boldsymbol{\vartheta}_{S}, f_{1} ; 33}^{\star} & :=\boldsymbol{\Gamma}_{\boldsymbol{\vartheta}_{S}, f_{1} ; 33}-\boldsymbol{\Gamma}_{\boldsymbol{\vartheta}_{S}, f_{1} ; 32} \Gamma_{\boldsymbol{\vartheta}_{S}, f_{1} ; 22}^{-1} \boldsymbol{\Gamma}_{\boldsymbol{\vartheta}_{S}, f_{1} ; 32}^{\prime} \\
& =\frac{\mathcal{J}_{k}\left(f_{1}\right)}{4 k(k+2)} \mathbf{M}_{S}^{\mathbf{V}_{S}}\left(\mathbf{V}_{S}^{\otimes 2}\right)^{-1 / 2}\left[\mathbf{I}_{k^{2}}+\mathbf{K}_{k}-\frac{2}{k} \mathbf{J}_{k}\right]\left(\mathbf{V}_{S}^{\otimes 2}\right)^{-1 / 2}\left(\mathbf{M}_{S}^{\mathbf{V}_{S}}\right)^{\prime}
\end{aligned}
$$

that is, the asymptotic covariance matrix, under $P_{\vartheta_{S}, f_{1}}^{n}$, of the $f_{1}$-efficient central sequence for shape

$$
\begin{aligned}
\boldsymbol{\Delta}_{\vartheta_{S}, f_{1} ; 3}^{\star n} & :=\boldsymbol{\Delta}_{\vartheta_{S}, f_{1} ; 3}^{n}-\boldsymbol{\Gamma}_{\vartheta_{S}, f_{1} ; 32} \Gamma_{\vartheta_{S}, f_{1} ; 22}^{-1} \Delta_{\vartheta_{S}, f_{1} ; 2}^{n} \\
& =\frac{1}{2 \sqrt{n}} \mathbf{M}_{S}^{\mathbf{V}_{S}}\left(\mathbf{V}_{S}^{\otimes 2}\right)^{-1 / 2} \sum_{i=1}^{n} \varphi_{f_{1}}\left(\frac{d_{i}}{\sigma_{S}}\right) \frac{d_{i}}{\sigma_{S}} \operatorname{vec}\left(\mathbf{U}_{i} \mathbf{U}_{i}^{\prime}-\frac{1}{k} \mathbf{I}_{k}\right)
\end{aligned}
$$

as explained in a general parametric setup in, e.g, Section 2.4 of Bickel et al. (1993) locally optimal inference on shape - under unspecified $\sigma_{S}^{2}$ - should be based on $\boldsymbol{\Delta}_{\vartheta_{S}, f_{1} ; 3}^{\star n}$.

Note that, unlike the original central sequence for shape in (5), $\Delta_{\vartheta_{S}, f_{1} ; 3}^{\star n}$ remains centered under any $P_{\vartheta_{S}, g_{1}}^{n}, g_{1} \neq f_{1}$. Actually, letting $K_{f_{1}}(u)=\varphi_{f_{1}}\left(\tilde{F}_{1 k}^{-1}(u)\right) \times \tilde{F}_{1 k}^{-1}(u)$ $(u \in(0,1))$, where $\tilde{F}_{1 k}$ stands for the cdf associated with the pdf $\tilde{f}_{1 k}$, and denoting by $R_{i}=R_{i}\left(\boldsymbol{\theta}, \mathbf{V}_{S}\right)$ the rank of $d_{i}=d\left(\mathbf{X}_{i}, \boldsymbol{\theta} ; \mathbf{V}_{S}\right)$ among $d_{1}, \ldots, d_{n}$, a trivial extension of the proof of Lemma 4.1 in Hallin and Paindaveine (2006a) (which is restricted to $S(\boldsymbol{\Sigma})=\Sigma_{11}$ ) yields that, under $P_{\vartheta_{S}, f_{1}}^{n}$, as $n \rightarrow \infty$,

$$
\Delta_{\vartheta_{S}, f_{1} ; 3}^{\star n}=\frac{1}{2 \sqrt{n}} \mathbf{M}_{S}^{\mathbf{V}_{S}}\left(\mathbf{V}_{S}^{\otimes 2}\right)^{-1 / 2} \sum_{i=1}^{n} K_{f_{1}}\left(\frac{R_{i}}{n+1}\right) \operatorname{vec}\left(\mathbf{U}_{i} \mathbf{U}_{i}^{\prime}-\frac{1}{k} \mathbf{I}_{k}\right)+o_{P}(1)
$$


which shows that $\Delta_{\vartheta_{S}, f_{1} ; 3}^{\star n}$ admits an asymptotically equivalent version based on the ranks $R_{i}$ and the multivariate signs $\mathbf{U}_{i}$. This asymptotic equivalence, along with the invariance properties of the families $\mathcal{P}_{S ; \vartheta_{S}}^{n}=\left\{P_{\vartheta_{S}, g_{1}}^{n}: g_{1} \in \mathcal{F}\right\}$ (see, for $S(\boldsymbol{\Sigma})=\Sigma_{11}$, Section 4.1 in Hallin and Paindaveine 2006a) and a general result by Hallin and Werker (2003), shows that the (semiparametrically) optimal performance (at $P_{\vartheta_{S}, f_{1}}^{n}$ ), when performing inference on shape in $\mathcal{P}_{S}^{n}=\left\{P_{\vartheta_{S}, g_{1}}^{n}: \boldsymbol{\vartheta}_{S} \in \boldsymbol{\Theta}_{S}, g_{1} \in \mathcal{F}\right\}$ concides with the optimal performance achievable in the parametric model $\mathcal{P}_{S ; f_{1}}^{n}=\left\{P_{\boldsymbol{\vartheta}_{S}, f_{1}}^{n}: \boldsymbol{\vartheta}_{S} \in \boldsymbol{\Theta}_{S}\right\}$ (as characterized by the efficient information matrix $\Gamma_{\vartheta_{S}, f_{1} ; 33}^{\star}$ in (8)). This confirms the adaptivity result first obtained in Example 4 of Bickel (1982), where it is shown that the non-specification of $f_{1}$ has no cost when estimating the inverse shape matrix $\mathbf{V}^{-1}:=\boldsymbol{\Sigma}^{-1} /\left(\operatorname{tr} \boldsymbol{\Sigma}^{-1}\right)$; note that although this adaptivity result restricts to what is called there a "most general" normalization (the one based on the trace), its proof actually holds for an arbitrary scale functional $S$.

Summing up, when estimating the shape $\mathbf{V}_{S}$ in $\mathcal{P}_{S}^{n}=\left\{P_{\vartheta_{S}, g_{1}}^{n}: \boldsymbol{\vartheta}_{S} \in \boldsymbol{\Theta}_{S}, g_{1} \in \mathcal{F}\right\}$, the non-specification of $\sigma_{S}^{2}$ alone is responsible for a loss of efficiency (as already mentioned, the non-specification of $\boldsymbol{\theta}$ does not play any role). This property - call it Bickel adaptivityactually holds for an arbitrary scale functional $S$. In this paper, we consider a stronger adaptivity concept - full adaptivity, say - under which $\boldsymbol{\theta}, \sigma_{S}^{2}$ and $f_{1}$ (rather than $f_{1}$ alone) lie in the nuisance space of the semiparametric model. The next section shows that full adaptivity holds for one and only one scale functional $S$, which therefore can be considered canonical.

\section{A canonical definition of shape.}

We are now ready to state the main result of the paper, which shows that there exists a unique scale functional $S$ (thus a unique definition of the shape $\mathbf{V}_{S}$ ) for which the nonspecification of $\sigma_{S}^{2}$ does not cause any loss of efficiency when performing inference on $\mathbf{V}_{S}$ (the loss of efficiency, for any fixed $S$, is studied in Hallin and Paindaveine 2006b, both for point estimation and hypothesis testing), hence, for which inference on $\mathbf{V}_{S}$ in $\mathcal{P}_{S}^{n}$ and $\mathcal{P}_{S ; \sigma_{S}^{2}, f_{1}}^{n}$ (equivalently, $\mathcal{P}_{S ; \boldsymbol{\theta}, \sigma_{S}^{2}, f_{1}}^{n}$ ) yields the same optimal performance.

Theorem 3.1 Let Assumptions (A1) and (A2) hold. Then $\boldsymbol{\Gamma}_{\boldsymbol{\vartheta}_{S}, f_{1} ; 32}=\mathbf{0}$ for all $\boldsymbol{\vartheta}_{S} \in \boldsymbol{\Theta}_{S}$ iff $S=S_{d}$, where $S_{d}(\boldsymbol{\Sigma}):=|\boldsymbol{\Sigma}|^{1 / k}$.

The decomposition of scatter into scale and shape through the functional $S_{d}$ is thus the only one that guarantees (a) mutual (asymptotic) orthogonality of the scale and shape parts of the central sequence (hence, independence in the asymptotic multinormal distribution), and, consequently, (b) full adaptivity (see above) in the estimation of shape. This finding strongly pleads in favor of the determinant-based definition of shape which, with its block-diagonal information matrix, is also more convenient from the point of view of statistical inference.

For this canonical parametrization, the shape parts of the central sequence and Fisher information matrix take the simple form

$$
\boldsymbol{\Delta}_{\vartheta_{S}, f_{1} ; 3}^{n}=\frac{1}{2 \sqrt{n}} \mathbf{M}_{S}^{\mathbf{V}_{S}}\left(\mathbf{V}_{S}^{\otimes 2}\right)^{-1 / 2} \sum_{i=1}^{n} \varphi_{f_{1}}\left(\frac{d_{i}}{\sigma_{S}}\right) \frac{d_{i}}{\sigma_{S}} \operatorname{vec}\left(\mathbf{U}_{i} \mathbf{U}_{i}^{\prime}\right)=\Delta_{\vartheta_{S}, f_{1} ; 3}^{\star n},
$$


and

$$
\Gamma_{\vartheta_{S}, f_{1} ; 33}=\frac{\mathcal{J}_{k}\left(f_{1}\right)}{4 k(k+2)} \mathbf{M}_{S}^{\mathbf{V}_{S}}\left[\mathbf{I}_{k^{2}}+\mathbf{K}_{k}\right]\left(\mathbf{V}_{S}^{\otimes 2}\right)^{-1}\left(\mathbf{M}_{S}^{\mathbf{V}_{S}}\right)^{\prime}=\Gamma_{\vartheta_{S}, f_{1} ; 33}^{\star}
$$

(note indeed that the proof of Theorem 3.1 shows that, for the canonical parametrization, $\mathbf{M}_{S}^{\mathbf{V}_{S}}\left(\operatorname{vec} \mathbf{V}_{S}^{-1}\right)=\mathbf{0}$ for all $\mathbf{V}_{S} \in \mathcal{V}_{k}^{S}$, which entails that $\left.\mathbf{M}_{S}^{\mathbf{V}_{S}}\left(\mathbf{V}_{S}^{\otimes 2}\right)^{-1 / 2} \mathbf{J}_{k}=\mathbf{0}\right)$. Theorem 3.1 shows that the determinant-based definition of scale/shape is the only one for which parametric and semiparametric efficiency bounds do coincide (hence, no other choice of $S$ is such that $\Delta_{\vartheta_{S}, f_{1} ; 3}^{\star n}$ and $\Delta_{\vartheta_{S}, f_{1} ; 3}^{n}$ are equal up to $o_{P}(1)$ terms). Also note that, since $\mathcal{J}_{k}\left(f_{1}\right)=k(k+2)$ at the multinormal, the canonical parametrization of shape is also the only one for which the information matrix for shape (either in its original or efficient version) in (10) is at any $f_{1}$ proportional to the parametric information matrix (7) at the multinormal.

We end this section by stressing that the canonical definition of scale/shape is not only relevant for problems involving scale or shape alone, but also for problems on scatter matrices. As an illustration, consider the two-sample problem for covariance matrices (in the rest of this section, we assume that every distribution has finite second-order moments). More precisely, assume that the $n:=n_{1}+n_{2}$ observations $\mathbf{X}_{i j}, j=1, \ldots, n_{i}, i=1,2$ are mutually independent random vectors admitting the pdf in (2) with location $\boldsymbol{\theta}_{i}$, scatter $\boldsymbol{\Sigma}_{i}$, and standardized radial density $f_{1 i}$; here, we rather standardize $f_{1 i}, i=1,2$ in such a way that $\boldsymbol{\Sigma}_{i}$ is the population covariance matrix of $\mathbf{X}_{i j}$. In this setup, we consider the problem of testing the null hypothesis $\mathcal{H}_{0}: \Sigma_{1}=\Sigma_{2}$ of covariance homogeneity.

For any scale functional $S$ satisfying Assumption (A1), the null $\mathcal{H}_{0}$ can be rewritten as $\mathcal{H}_{0}=\mathcal{H}_{0}^{\text {scale }} \cap \mathcal{H}_{0}^{\text {shape }}$, where $\mathcal{H}_{0}^{\text {scale }}: S\left(\boldsymbol{\Sigma}_{1}\right)=S\left(\boldsymbol{\Sigma}_{2}\right)\left(\right.$ resp., $\left.\mathcal{H}_{0}^{\text {shape }}: \boldsymbol{\Sigma}_{1} / S\left(\boldsymbol{\Sigma}_{1}\right)=\boldsymbol{\Sigma}_{2} / S\left(\boldsymbol{\Sigma}_{2}\right)\right)$ is the hypothesis of scale (resp., shape) homogeneity. Deviations from the null $\mathcal{H}_{0}$ can result from a violation of $\mathcal{H}_{0}^{\text {scale }}$, of $\mathcal{H}_{0}^{\text {shape }}$, or of both $\mathcal{H}_{0}^{\text {scale }}$ and $\mathcal{H}_{0}^{\text {shape }}$. Standard tests (such as the Gaussian LRT; see, e.g., Bilodeau and Brenner 1999, page 121) do not take into account the decomposition of covariance matrices into scale and shape, hence do not provide any insight into the reasons why a possible rejection occurs.

On the contrary, if covariance matrices are decomposed into scale and shape, Le Cam's methodology naturally leads to test statistics of the form $Q=Q^{\text {scale }}+Q^{\text {shape }}$, where, irrespective of the choice of $S, Q^{\text {scale }}$ and $Q^{\text {shape }}$ are (under the null) asymptotically independent chi-square random variables (with 1 and $K$ degrees of freedom, respectively), whose p-values provide an evaluation of the respective deviations from $\mathcal{H}_{0}^{\text {scale }}$ and $\mathcal{H}_{0}^{\text {shape }}$ in an eventual rejection of $\mathcal{H}_{0}$, hence an interesting insight into the reasons why rejection occurs. It is crucial, however, to note that the canonical definition of scale/shape in Theorem 3.1 is the only one for which $Q^{\text {scale }}$ and $Q^{\text {shape }}$ are locally and asymptotically (parametrically) optimal test statistics for $\mathcal{H}_{0}$ against scale and shape subalternatives, respectively. We refer to Hallin and Paindaveine (2008a,b) for more details.

\section{Proofs.}

In this final section, we prove Theorems 2.1 and 3.1.

For any $S$ satisfying Assumption (A1), consider the mapping $V_{11}^{S}$ : vech $\mathcal{S}^{k} \rightarrow \mathbb{R}$ defined by $S\left(\left(V_{11}^{S}\left(\operatorname{vech}^{\circ}\right) \text {, }(\operatorname{vech} \mathbf{V})^{\prime}\right)^{\prime}\right)=1$, the existence of which-locally around any $\mathbf{V}_{S} \in \mathcal{V}_{k}^{S}$ is guaranteed by Assumption (A1) and the implicit function Theorem. We then start with the following lemma. 
Lemma 4.1 Let Assumption (A1) hold and fix $\mathbf{V}_{S} \in \mathcal{V}_{k}^{S}$. Let also $\mathbf{P}_{k}$ be the $(K+1) \times k^{2}$ matrix such that $\mathbf{P}_{k}^{\prime}($ vech $\mathbf{v})=\operatorname{vec} \mathbf{v}$ for any symmetric $k \times k$ matrix $\mathbf{v}$. Then, $\mathbf{M}_{S}^{\mathbf{V}_{S}}=$ $\left(\nabla V_{11}^{S}\left(\operatorname{vech} \mathbf{V}_{S}\right) \vdots \mathbf{I}_{K}\right) \mathbf{P}_{k}$.

Proof of Lemma 4.1. Differentiating (at vech $\left.\mathbf{V}_{S}\right)$ both sides of $S\left(\left(V_{11}^{S}(\right.\right.$ vech $\mathbf{V})$, $\left.\left.\left(\text { vech }^{\circ}\right)^{\prime}\right)^{\prime}\right)=1$ with respect to vech $\mathbf{V}$, we obtain

$$
\nabla V_{11}^{S}\left(\operatorname{vech} \mathbf{V}_{S}\right)=-\frac{\nabla_{2} S\left(\operatorname{vech} \mathbf{V}_{S}\right)}{\nabla_{1} S\left(\operatorname{vech} \mathbf{V}_{S}\right)}
$$

where $\nabla S\left(\operatorname{vech} \mathbf{V}_{S}\right)=\left(\nabla_{1} S\left(\operatorname{vech} \mathbf{V}_{S}\right),\left(\nabla_{2} S\left(\operatorname{vech} \mathbf{V}_{S}\right)\right)^{\prime}\right)^{\prime} \in \mathbb{R} \times \mathbb{R}^{K}$. Now, $\mathbf{M}_{S}^{\mathbf{V}_{S}}$ is such that $\left(\mathbf{M}_{S}^{\mathbf{V}_{S}}\right)^{\prime}($ vech $\mathbf{v})=(\operatorname{vec} \mathbf{v})$ for any symmetric $k \times k$ matrix $\mathbf{v}=\left(v_{i j}\right)$ satisfying $\left(\nabla S\left(\operatorname{vech} \mathbf{V}_{S}\right)\right)^{\prime}(\operatorname{vech} \mathbf{v})=\left(\nabla_{1} S\left(\operatorname{vech} \mathbf{V}_{S}\right)\right) \times v_{11}+\left(\nabla_{2} S\left(\operatorname{vech} \mathbf{V}_{S}\right)\right)^{\prime}(\operatorname{vech} \mathbf{v})=0$, that is, any symmetric $\mathbf{v}$ satisfying $\left(\nabla V_{11}^{S}\left(\text { vech } \mathbf{V}_{S}\right)\right)^{\prime}($ vech $\mathbf{v})=v_{11}$ (see (11)). The result follows since, for any such $\mathbf{v}$, one also has $\left(\left(\nabla V_{11}^{S}\left(\operatorname{vech}_{\mathbf{V}_{S}}\right) \vdots \mathbf{I}_{K}\right) \mathbf{P}_{k}\right)^{\prime}($ vech $\mathbf{v})=\mathbf{P}_{k}^{\prime}\left(v_{11},(\text { vech } \mathbf{v})^{\prime}\right)^{\prime}=$ $\mathbf{P}_{k}^{\prime}(\operatorname{vech} \mathbf{v})=\operatorname{vec} \mathbf{v}$.

Proof of Theorem 2.1. As shown in the proof of Proposition 2.1 in Hallin and Paindaveine (2006a), the family $\mathcal{P}_{f_{1}}^{n}:=\left\{P_{\boldsymbol{\xi}, f_{1}}^{n}=P_{\boldsymbol{\theta}, \boldsymbol{\Sigma}, f_{1}}^{n}: \boldsymbol{\xi}=\left(\boldsymbol{\theta}^{\prime},(\operatorname{vech} \boldsymbol{\Sigma})^{\prime}\right)^{\prime} \in \boldsymbol{\Xi}:=\right.$ $\mathbb{R}^{k} \times\left(\right.$ vech $\left.\left.\mathcal{S}_{k}\right)\right\}$, under Assumption (A2), is LAN. Now, for any scale functional $S$ satisfying Assumption (A1), consider the function $h^{S}: \boldsymbol{\Theta}_{S} \rightarrow \boldsymbol{\Xi}$ that maps $\boldsymbol{\vartheta}_{S}$ onto the corresponding value of $\boldsymbol{\xi}$, namely onto $\boldsymbol{\xi}=h^{S}\left(\boldsymbol{\vartheta}_{S}\right)=\left(\boldsymbol{\theta}^{\prime}, \sigma_{S}^{2}\left(\operatorname{vech} \mathbf{V}_{S}\right)^{\prime}\right)^{\prime}=\left(\boldsymbol{\theta}^{\prime}, \sigma_{S}^{2} V_{11}^{S}\left(\operatorname{vech} \mathbf{V}_{S}\right), \sigma_{S}^{2}\left(\operatorname{vech} \mathbf{V}_{S}\right)^{\prime}\right)^{\prime}$. Since $h^{S}$ is a diffeomorphism, $\mathcal{P}_{S ; f_{1}}^{n}=\left\{P_{\boldsymbol{\vartheta}_{S}, f_{1}}^{n}: \boldsymbol{\vartheta}_{S} \in \boldsymbol{\Theta}_{S}\right\}$ is also LAN, and the corresponding central sequence is given by

$$
\boldsymbol{\Delta}_{\boldsymbol{\vartheta}_{S}, f_{1}}^{n}=\left(D h^{S}\left(\boldsymbol{\vartheta}_{S}\right)\right)^{\prime} \bar{\Delta}_{h\left(\boldsymbol{\vartheta}_{S}\right), f_{1}}^{n},
$$

where

$$
D h^{S}\left(\boldsymbol{\vartheta}_{S}\right)=\left(\begin{array}{ccc}
\mathbf{I}_{k} & \mathbf{0} & \mathbf{0} \\
\mathbf{0} & V_{11}^{S}\left(\operatorname{oech~}_{S}\right) & \sigma_{S}^{2}\left(\nabla V_{11}^{S}\left(\operatorname{voch} \mathbf{V}_{S}\right)\right)^{\prime} \\
\mathbf{0} & \operatorname{vech}_{S} \mathbf{V}_{S} & \sigma_{S}^{2} \mathbf{I}_{K}
\end{array}\right)
$$

is the Jacobian matrix of $h^{S}$ at $\boldsymbol{\vartheta}_{S}$ and where, letting $d_{i}(\boldsymbol{\xi})=d\left(\mathbf{X}_{i}, \boldsymbol{\theta} ; \boldsymbol{\Sigma}\right)$ and $\mathbf{U}_{i}(\boldsymbol{\xi})=$ $\boldsymbol{\Sigma}^{-1 / 2}\left(\mathbf{X}_{i}-\boldsymbol{\theta}\right) / d_{i}(\boldsymbol{\xi})$ (where $\boldsymbol{\Sigma}^{1 / 2}$ denotes the symmetric root of $\boldsymbol{\Sigma}$ ),

$$
\bar{\Delta}_{\boldsymbol{\xi}, f_{1}}^{n}:=\left(\begin{array}{c}
\frac{1}{\sqrt{n}} \sum_{i=1}^{n} \varphi_{f_{1}}\left(d_{i}(\boldsymbol{\xi})\right) \boldsymbol{\Sigma}^{-1 / 2} \mathbf{U}_{i}(\boldsymbol{\xi}) \\
\frac{1}{2 \sqrt{n}} \mathbf{P}_{k}\left(\boldsymbol{\Sigma}^{\otimes 2}\right)^{-1 / 2} \sum_{i=1}^{n} \operatorname{vec}\left(\varphi_{f_{1}}\left(d_{i}(\boldsymbol{\xi})\right) d_{i}(\boldsymbol{\xi}) \mathbf{U}_{i}(\boldsymbol{\xi}) \mathbf{U}_{i}^{\prime}(\boldsymbol{\xi})-\mathbf{I}_{k}\right)
\end{array}\right)
$$

is the central sequence in the LAN family $\mathcal{P}_{f_{1}}^{n}=\left\{P_{\xi, f_{1}}^{n}: \xi \in \Xi\right\}$ (see the proof of Proposition 2.1 in Hallin and Paindaveine 2006a). The result then readily follows from (12), since (i) the scale part $\boldsymbol{\Delta}_{\vartheta_{S}, f_{1} ; 2}^{n}$ of $\boldsymbol{\Delta}_{\vartheta_{S}, f_{1}}^{n}$ can be written as in (4) by noting that (vech $\left.\mathbf{V}_{S}\right)^{\prime} \mathbf{P}_{k}$ $\left(\mathbf{V}_{S}^{\otimes 2}\right)^{-1 / 2} \operatorname{vec} \mathbf{v}=(\operatorname{tr} \mathbf{v})$ for any $k \times k$ symmetric matrix $\mathbf{v}$, and since (ii) the shape part $\Delta_{\vartheta_{S}, f_{1} ; 3}^{n}$ of $\Delta_{\vartheta_{S}, f_{1}}^{n}$ can be directly put under the form (5) by using Lemma 4.1.

Now, for any $\boldsymbol{\Sigma} \in \mathcal{S}_{k}$ and $S$ satisfying Assumption (A1), define $\mathbf{C}_{S}^{\Sigma}:=\mathbf{C}_{S, k}^{\Sigma}$ as the upper-triangular $k \times k$ matrix such that vech $\mathbf{C}_{S}^{\boldsymbol{\Sigma}}:=\nabla S(\operatorname{vech} \boldsymbol{\Sigma})$, and let $\mathbf{D}_{S}^{\boldsymbol{\Sigma}}:=\left(\mathbf{C}_{S}^{\boldsymbol{\Sigma}}+\right.$ $\left.\left(\mathbf{C}_{S}^{\boldsymbol{\Sigma}}\right)^{\prime}\right) / 2$. Clearly, $\left(\operatorname{vec} \mathbf{D}_{S}^{\boldsymbol{\Sigma}}\right)^{\prime}(\operatorname{vec} \mathbf{v})=\left(\operatorname{vech} \mathbf{C}_{S}^{\boldsymbol{\Sigma}}\right)^{\prime}(\operatorname{vech} \mathbf{v})$ for any symmetric $k \times k$ matrix $\mathbf{v}$. The matrix $\mathbf{M}_{S}^{\Sigma}$ is then such that $\left(\mathbf{M}_{S}^{\Sigma}\right)^{\prime}($ vech $\mathbf{v})=($ vec $\mathbf{v})$ for any symmetric $k \times k$ 
matrix v satisfying $\left(\operatorname{vec} \mathbf{D}_{S}^{\boldsymbol{\Sigma}}\right)^{\prime}(\operatorname{vec} \mathbf{v})=0$ (equivalently, satisfying $\operatorname{tr}\left(\mathbf{D}_{S}^{\boldsymbol{\Sigma}} \mathbf{v}\right)=0$ ). For $S(\boldsymbol{\Sigma})=\Sigma_{11}, S(\boldsymbol{\Sigma})=(\operatorname{tr} \boldsymbol{\Sigma}) / k$, and $S(\boldsymbol{\Sigma})=|\boldsymbol{\Sigma}|^{1 / k}$, one has $\mathbf{D}_{S}^{\boldsymbol{\Sigma}}=\mathbf{e}_{1} \mathbf{e}_{1}^{\prime}, \mathbf{D}_{S}^{\boldsymbol{\Sigma}}=\frac{1}{k} \mathbf{I}_{k}$, and $\mathbf{D}_{S}^{\boldsymbol{\Sigma}}=\frac{1}{k}|\boldsymbol{\Sigma}|^{1 / k} \boldsymbol{\Sigma}^{-1}$, respectively. The following result states some important properties of $\mathbf{M}_{S}^{\Sigma}$ and $\mathbf{D}_{S}^{\Sigma}$, which are needed in the proof of Theorem 3.1.

Lemma 4.2 Let Assumption (A1) hold and fix $\boldsymbol{\Sigma} \in \mathcal{S}_{k}$. Then, (i) $\mathbf{D}_{S}^{\boldsymbol{\Sigma}}=\mathbf{D}_{S}^{\lambda \boldsymbol{\Sigma}}$ for all $\lambda>0$; (ii) $S(\boldsymbol{\Sigma})=\operatorname{tr}\left(\mathbf{D}_{S}^{\boldsymbol{\Sigma}} \boldsymbol{\Sigma}\right)$; (iii) letting $\boldsymbol{\Sigma}_{\lambda}:=(1-\lambda) \mathbf{I}_{k}+\lambda \boldsymbol{\Sigma}, S(\boldsymbol{\Sigma})=\exp \left[\int_{0}^{1} \operatorname{tr}((\boldsymbol{\Sigma}-\right.$ $\left.\left.\left.\mathbf{I}_{k}\right) \mathbf{D}_{S}^{\boldsymbol{\Sigma}_{\lambda}} / S\left(\boldsymbol{\Sigma}_{\lambda}\right)\right) d \lambda\right]$; (iv) $\mathbf{M}_{S}^{\boldsymbol{\Sigma}}$ has (maximal) rank $K$; (v) denoting by ker $\mathbf{A}$ the null space of a matrix $\mathbf{A},\left(\operatorname{ker} \mathbf{M}_{S}^{\boldsymbol{\Sigma}}\right) \cap\left(\operatorname{vec} \mathcal{S}_{k}\right)=\left\{\lambda\left(\operatorname{vec} \mathbf{D}_{S}^{\boldsymbol{\Sigma}}\right): \lambda>0\right\}$.

Proof of Lemma 4.2. (i) The claim readily follows from the definition of $\mathbf{D}_{S}^{\Sigma}$ and the 1-homogeneity of $S$, which implies that the gradient of $S$ is constant along half lines of the form $\{\lambda \boldsymbol{\Sigma}: \lambda>0\}$. (ii) By taking derivatives at $\lambda=1$ of both sides of the equality $\lambda S(\operatorname{vech} \boldsymbol{\Sigma})=S(\lambda(\operatorname{vech} \boldsymbol{\Sigma}))$, one obtains that $S(\boldsymbol{\Sigma})=\left(\operatorname{vech} \mathbf{C}_{S}^{\boldsymbol{\Sigma}}\right)^{\prime}(\operatorname{vech} \boldsymbol{\Sigma})=$ $\left(\operatorname{vec} \mathbf{D}_{S}^{\boldsymbol{\Sigma}}\right)^{\prime}(\operatorname{vec} \boldsymbol{\Sigma})=\operatorname{tr}\left(\mathbf{D}_{S}^{\boldsymbol{\Sigma}} \boldsymbol{\Sigma}\right)$. (iii) The result follows by integrating between 0 and 1 the mapping $\lambda \mapsto d \log S\left(\boldsymbol{\Sigma}_{\lambda}\right) / d \lambda=\left(\operatorname{vech}\left(\boldsymbol{\Sigma}-\mathbf{I}_{k}\right)\right)^{\prime}\left(\operatorname{vech} \mathbf{C}_{S}^{\boldsymbol{\Sigma}_{\lambda}}\right) / S\left(\boldsymbol{\Sigma}_{\lambda}\right)=(\operatorname{vec}(\boldsymbol{\Sigma}-$ $\left.\left.\mathbf{I}_{k}\right)\right)^{\prime}\left(\operatorname{vec} \mathbf{D}_{S}^{\boldsymbol{\Sigma}_{\lambda}}\right) / S\left(\boldsymbol{\Sigma}_{\lambda}\right)=\operatorname{tr}\left(\left(\boldsymbol{\Sigma}-\mathbf{I}_{k}\right) \mathbf{D}_{S}^{\boldsymbol{\Sigma}_{\lambda}} / S\left(\boldsymbol{\Sigma}_{\lambda}\right)\right)$. (iv) It is clear from the definition of $\mathbf{M}_{S}^{\boldsymbol{\Sigma}}$ that $\left(\mathbf{M}_{S}^{\boldsymbol{\Sigma}}\right)^{\prime}$ maps the canonical basis of $\mathbb{R}^{K}$ into a collection of linearly independent vectors in $\mathbb{R}^{k^{2}}$. Hence, $\operatorname{rank}\left(\mathbf{M}_{S}^{\Sigma}\right)=K$. (v) It follows from the definition of $\mathbf{M}_{S}^{\Sigma}$ that, for any symmetric $k \times k$ matrix $\mathbf{v}$ satisfying $\left(\operatorname{vec} \mathbf{D}_{S}^{\boldsymbol{\Sigma}}\right)^{\prime}(\operatorname{vec} \mathbf{v})=0,\left(\operatorname{vec} \mathbf{D}_{S}^{\boldsymbol{\Sigma}}\right)^{\prime}\left(\mathbf{M}_{S}^{\boldsymbol{\Sigma}}\right)^{\prime}(\operatorname{vech} \mathbf{v})=0$. Since Assumption (A1) guarantees that $\left(\mathbf{D}_{S}^{\Sigma}\right)_{11}=\frac{\partial S}{\partial \Sigma_{11}}(\boldsymbol{\Sigma}) \neq 0$ for all $\boldsymbol{\Sigma} \in \mathcal{S}_{k}$, this entails that $\mathbf{M}_{S}^{\Sigma}\left(\operatorname{vec} \mathbf{D}_{S}^{\boldsymbol{\Sigma}}\right)=\mathbf{0}$. Now, the proof of (iv) shows that the restriction ( $L$, say) to (vec $\mathcal{S}_{k}$ ) of the linear mapping from $\mathbb{R}^{k^{2}}$ to $\mathbb{R}^{K}$ with matrix $\mathbf{M}_{S}^{\Sigma}$ has rank $K$. Hence, the null space of $L$ has dimension 1, which establishes the result.

We are now able to prove Theorem 3.1.

Proof of Theorem 3.1. Assume that $\boldsymbol{\Gamma}_{\boldsymbol{\vartheta}_{S}, f_{1} ; 32}=\mathbf{0}$ for all $\boldsymbol{\vartheta}_{S} \in \boldsymbol{\Theta}_{S}$. Since Assumption (A2) guarantees that $\mathcal{J}_{k}\left(f_{1}\right)>k^{2}$ (see Hallin and Paindaveine 2006a), we must have that $\mathbf{M}_{S}^{\mathbf{V}_{S}}\left(\operatorname{vec} \mathbf{V}_{S}^{-1}\right)=\mathbf{0}$ for all $\mathbf{V}_{S} \in \mathcal{V}_{k}^{S}$. Lemma $4.2(\mathrm{v})$ shows that, for all $\mathbf{V}_{S} \in \mathcal{V}_{k}^{S}$, there exists some $\lambda\left(\mathbf{V}_{S}\right) \neq 0$ such that $\mathbf{V}_{S}^{-1}=\lambda\left(\mathbf{V}_{S}\right) \mathbf{D}_{S}^{\mathbf{V}_{S}}$. Lemma 4.2(ii) then yields that $1=S\left(\mathbf{V}_{S}\right)=\operatorname{tr}\left(\left(\lambda\left(\mathbf{V}_{S}\right)\right)^{-1} \mathbf{I}_{k}\right)=\left(\lambda\left(\mathbf{V}_{S}\right)\right)^{-1} k$, so that $\lambda\left(\mathbf{V}_{S}\right)=k$ for all $\mathbf{V}_{S} \in \mathcal{V}_{k}^{S}$. Hence, Lemma 4.2(i) entails that, for any $\boldsymbol{\Sigma} \in \mathcal{S}_{k}, \mathbf{D}_{S}^{\boldsymbol{\Sigma}} / S(\boldsymbol{\Sigma})=\mathbf{D}_{S}^{\boldsymbol{\Sigma} / S(\boldsymbol{\Sigma})} / S(\boldsymbol{\Sigma})=$ $\frac{1}{k}(\boldsymbol{\Sigma} / S(\boldsymbol{\Sigma}))^{-1} / S(\boldsymbol{\Sigma})=\frac{1}{k} \boldsymbol{\Sigma}^{-1}$. Since we also have that $\mathbf{D}_{S_{d}}^{\boldsymbol{\Sigma}} / S_{d}(\boldsymbol{\Sigma})=\frac{1}{k} \boldsymbol{\Sigma}^{-1}$ (see the paragraph below the proof of Theorem 2.1), Lemma 4.2(iii) yields that $S(\boldsymbol{\Sigma})=S_{d}(\boldsymbol{\Sigma})$ for any $\Sigma \in \mathcal{S}_{k}$, which establishes the result.

\section{References}

[1] Bickel, P.J. (1982). On adaptive estimation, Ann. Statist. 10, 647-671.

[2] Bickel, P.J. , C.A.J. Klaassen, Y. Ritov and J.A. Wellner (1993). Efficient and Adaptive Estimation for Semiparametric Models (Johns Hopkins University Press, Baltimore).

[3] Bilodeau, M. and D. Brenner (1999). Theory of Multivariate Analysis. SpringerVerlag, New York. 
[4] Croux, C. and G. Haesbroeck (2000). Principal component analysis based on robust estimators of the covariance or correlation matrix : influence functions and efficiencies, Biometrika 87, 603-618.

[5] Dümbgen, L., and D.E. Tyler (2005). On the breakdown properties of some multivariate M-Functionals, Scand. J. Statist. 32, 247-264.

[6] Dümbgen, L. (1998). On Tyler's M-functional of scatter in high dimension, Ann. Inst. Statist. Math. 50, 471-491.

[7] Hallin, M. and D. Paindaveine (2006a). Semiparametrically efficient rank-based inference for shape. I. Optimal rank-based tests for sphericity, Ann. Statist. 34, 2707-2756.

[8] Hallin, M. and D. Paindaveine (2006b). Parametric and semiparametric inference for shape: the role of the scale functional, Statist. Decisions 24, 327-350.

[9] Hallin, M., H. Oja, and D. Paindaveine (2006). Semiparametrically efficient rankbased inference for shape. II. Optimal R-estimation of shape, Ann. Statist. 34, 27572789.

[10] Hallin, M. and D. Paindaveine (2008a). Optimal rank-based tests for homogeneity of scatter, Ann. Statist., to appear.

[11] Hallin, M. and D. Paindaveine (2008b). Optimal tests for homogeneity of covariance, scale, and shape. Submitted.

[12] Hallin, M., and B.J.M. Werker (2003). Semiparametric efficiency, distributionfreeness, and invariance, Bernoulli 9, 137-165.

[13] Hettmansperger, T.P. and R.H. Randles (2002). A practical affine equivariant multivariate median, Biometrika 89, 851-860.

[14] Le Cam, L. (1986). Asymptotic Methods in Statistical Decision Theory (SpringerVerlag, New York).

[15] Maronna, R., D. Martin, and V. Yohai (2006). Robust Statistics. Theory and Methods (Wiley, New York).

[16] Ollila, E., T.P. Hettmansperger, and H. Oja (2004). Affine equivariant multivariate sign methods. Preprint, University of Jyväskylä.

[17] Randles, R. H. (2000). A simpler, affine-invariant, multivariate, distribution-free sign test, J. Amer. Statist. Assoc. 95, 1263-1268.

[18] Salibian-Barrera, M., S. Van Aelst, and G. Willems (2006). Principal components analysis based on multivariate MM-estimators with fast and robust bootstrap, $J$. Amer. Statist. Assoc. 101, 1198-1211.

[19] Taskinen, S., C. Croux, A. Kankainen, E. Ollila, and H. Oja (2006). Influence functions and efficiencies of the canonical correlation and vector estimates based on scatter and shape matrices, J. Multivariate Anal. 97, 359-384.

[20] Tatsuoka, K.S., and D.E. Tyler (2000). On the uniqueness of S-Functionals and Mfunctionals under nonelliptical distributions, Ann. Statist. 28, 1219-1243.

[21] Tyler, D.E. (1983). Robustness and efficiency properties of scatter matrices, Biometrika 70, 411-420.

[22] Tyler, D.E. (1987). A distribution-free M-estimator of multivariate scatter, Ann. Statist. 15, 234-251. 
[23] Zuo, Y. (2006). Robust location and scatter estimators in multivariate analysis, in: J. Fan and H.L. Koul, Eds, Frontiers of Statistics (in honor of Professor P.J. Bickel's 65th Birthday), Imperial College, pp. 467-490. 